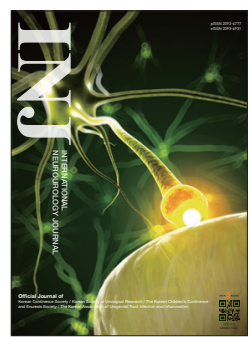

\title{
Pathomechanism of Interstitial Cystitis/Bladder Pain Syndrome and Mapping the Heterogeneity of Disease
}

\author{
Jia-Fong Jhang, Hann-Chorng Kuo \\ Department of Urology, Buddhist Tzu Chi General Hospital and Tzu Chi University, Hualien, Taiwan
}

\begin{abstract}
Interstitial cystitis/bladder pain syndrome (IC/BPS) is a heterogeneous syndrome which is usually characterized by urinary frequency, nocturia, and bladder pain. Several pathomechanisms have been proposed, including uroepithelial dysfunction, mast cell activation, neurogenic inflammation, autoimmunity, and occult urinary tract infections. It is possible that an inflammatory process alters regulation of urothelial homeostasis and results in dysfunction of the bladder epithelium. Different phenotypes of IC/BPS have been explored including Hunner and non-Hunner type IC, hypersensitive bladder, and bladder pain both with and without functional somatic syndrome. Different gene expressions have also been found in different IC phenotypes. Abnormal expressions of uroplakin, chondroitin sulfate and adhesive protein E-cadherin, tight junction protein zonula occludens- 1 in IC/BPS bladder suggest abnormal epithelial differentiation in this bladder disease. Analysis of inflammatory proteins, or cytokines in the urine or serum provides another diagnostic foundation forIC/BPS subtypes. The involvement of IC/BPS in systemic functional somatic syndrome and other pelvic organ diseases might also subdivide subtypes of IC/BPS. Chronic inflammation, increased urothelial apoptosis, and abnormal urothelial function are closely associated in IC bladders. This article reviews recent research on the pathomechanisms of IC, which might help us in mapping the heterogeneity of the disease.
\end{abstract}

Keywords: Lower Urinary Tract Symptoms; Cystitis; Bladder Pain; Biomarkers; Urotheliuim

- Conflict of Interest: No potential conflict of interest relevant to this article was reported.

\section{INTRODUCTION}

Interstitial cystitis/bladder pain syndrome (IC/BPS) is a chronic bladder condition which is characterized by bladder pain, urinary frequency, and nocturia. IC/BPS had been considered a progressive disease that may evolve from early to late stage. Patients might have an inflammatory reaction both in the bladder as well as in the central nervous system (CNS) after insults to the bladder, which produce early IC/BPS symptoms such as urinary frequency and nocturia. Patients may experience spontaneous symptom relief after the body's defense mechanism solves the inflammation in the bladder. However, the inflammation also might increase and cause permanent inflammatory imprinting in the bladder as well as in the CNS if the bladder insults persist. The current article reviews the recent research on the pathogenesis of IC/BPS with different clinical characteristics. Through histopathological study, urothelial barrier dysfunction and sensory protein expressions, and serum and urinary biomarker studies, we will combine clinical and basic data to map the heterogeneity of IC/BPS.

\section{CLINICAL CHARACTERISTICS WITH BLADDER HISTOPATHOLOGY IN IC/BPS}

Most urologists suggest the cause of IC/BPS might result from long-standing inflammation of the bladder [1]. However, the

Corresponding author: Hann-Chorng Kuo (iD http://orcid.org/0000-0001-7165-4771 Department of Urology, Buddhist Tzu Chi General Hospital, 707, Section 3, Chung Yang Road, Hualien, Taiwan

E-mail: hck@tzuchi.com.tw / Tel: +886-3-8561825, ext. 2117 / Fax: +886-3-8560794

Submitted: August 11, 2016 / Accepted after revision: September 30, 2016 
actual pathophysiology is still unclear. The most common clinical presentations of IC/PBS are bladder and lower abdominal pain, glomerulation hemorrhage after cystoscopic hydrodistention, or denudation or thinning of the urothelium. Clinical findings suggest bladder inflammation and urothelial dysfunction [2,3]. Urothelial dysfunction, chronic inflammation, impaired bladder circulation, neurogenic hyperactivity, and systemic functional disorders have been linked to IC/BPS and presumably interact in forming IC/BPS [4].

Denudation or thinning of the epithelium is the most common histopathological finding of IC/BPS [1]. Increased levels of urothelial cell apoptosis and decreased proliferation have been noted, suggesting an altered homeostasis in IC/BPS urothelium [5]. Abnormal expression of tight junction protein zonula occludens-1 (ZO-1), uroplakin and chondroitin sulfate have been found in IC/BPS bladders [6-8]. An increased antiproliferative factor and lower expression of interleukin (IL)-8 have been found in IC/BPS bladders, which may contribute to IC/BPS pathophysiology [9-12]. Altered urothelial differentiation might result in increased permeability and decreased protective func- tion of the bladder urothelium [13]. The increased urothelial permeability could result in a leaky urothelium and generate hypersensitive bladder symptoms [14]. Increased lymphocyte infiltration in the bladder interstitium and urothelial ulceration in chronic IC might limit the distention of the bladder, resulting in small functional bladder capacity and urination frequencyurgency symptoms. Increased sensory afferent activity including pain associated C-fibers in chronic IC will further cause bladder pain with a full bladder, especially in ulcer type IC/BPS [15].

Histopathological inflammation is a characteristic feature of the IC bladder [1]. Patients with Hunner ulcer IC/BPS show increased expression of $\mathrm{T}$ - and B-cell markers in the submucosa [16]. Overexpression of some proinflammatory genes has also been found in IC/BPS bladder [17-19]. It has been suggested that immunological reaction occurs in IC/BPS bladders [8]. A subset of IC/BPS patients might have an elevated serum IgE level [20]. However, no one pathogenic etiology can be adapted to fit all IC patients. It is apparent that IC is a heterogeneous syndrome resulting from different etiology and pathogenesis. Therefore, ex-

Table 1. Potential bladder tissue biomarkers for the diagnosis of IC/BPS

\begin{tabular}{|c|c|c|c|}
\hline Protein & Source & Evidence of activity & Reference \\
\hline $\begin{array}{l}\text { Pro-nociceptive } \\
\text { inflammatory proteins }\end{array}$ & Bladder & Increased expression of TRPV1, 2, 4, ASIC1, NGF, CXCL9, and TRPM2 in IC/BPS & 17 \\
\hline PDECGF/TP & Bladder & Highly associated with glomerulations during cystoscopic hydrodistention & 39 \\
\hline VEGF & Bladder & $\begin{array}{l}\text { Increased VEGF was associated with bladder inflammation and smaller functional } \\
\text { bladder capacity in IC/BPS }\end{array}$ & 41 \\
\hline HIF-1a & Bladder & $\begin{array}{l}\text { Increased expression of HIF-1 } 1 \alpha \text { and VEGF associated with glomerulations in } \\
\text { IC/BPS }\end{array}$ & 37 \\
\hline Apoptotic signaling molecules & Bladder & Bad, Bax, and cleaved caspase-3, were increased in the IC/BPS & 24 \\
\hline Urothelial barrier protein & Bladder & E-cadherin and ZO-1 expression was only decreased in IC/BPS & $5,8,29,30$ \\
\hline Differentiation related proteins & Bladder cell line & $\begin{array}{l}\text { A switch from a cytokeratin }(\mathrm{CK}) 13(\mathrm{lo}) / \mathrm{CK} 14(\mathrm{hi}) \text { to a CK13(hi)/CK14(lo) } \\
\text { phenotype, expression of claudin } 3,4 \text {, and } 5 \text { proteins, and induction of uroplakin } \\
\text { gene transcription }\end{array}$ & 6 \\
\hline Proteoglycan core proteins & Bladder & $\begin{array}{l}\text { Abnormal expression of keratin } 18 \text { and } 20 \text { and biglycan, decorin, perlecan, and } \\
\text { syndecan- } 1 \text { are found in IC/BPS }\end{array}$ & 8 \\
\hline Uroplakin III & Bladder & Over expression of uroplakin III-delta4 in nonulcerative type IC/BPS & 7 \\
\hline IL-8 & Bladder & Lower IL-8 expression levels in IC/BPS bladder & 12 \\
\hline NGF & Bladder & Increase of NGF level in IC/BPS & 58,59 \\
\hline Chemokines & Bladder & $\begin{array}{l}\text { Increased mRNA expression of CXCR3 binding chemokines (CXCL9, 10, and 11) } \\
\text { and TNFSF14 (LIGHT) in ulcer type IC/BPS }\end{array}$ & 19 \\
\hline
\end{tabular}

IC/BPS, interstitial cystitis/bladder pain syndrome; TRPV, TRP vanilloid; ASIC, acid sensing ion channel; NGF, nerve growth factor; CXCL9, chemokine (C-X-C motif) ligand 9; TRPM, TRP melastatin; PDECGF/TP, platelet derived endothelial cell growth factor/thymidine phosphorylase; VEGF, vascular endothelial growth factor; HIF-1 $\alpha$, hypoxia-inducible factor-1- $\alpha$; ZO-1, zonula occludens-1; IL, interleukin; CXCR, CXC chemokine receptor; TNFSF14, tumor necrosis factor ligand superfamily member 14. 
ploring the histopathological features in different inflammatory cells and bladder histopathology, and correlating these pathological findings with clinical characteristics might help us understand the possible pathomechanism of IC/BPS (Table 1).

\section{THE UROTHELIAL DYSFUNCTION IN IC/BPS}

The urothelium of the urinary bladder is considered to be a barrier between urine and the underlying bladder. Bladder surface mucus also plays an important role in this barrier function $[6,21]$. Failure of urothelial cytodifferentiation may contribute to the clinical features of IC/BPS [6]. Urothelial barrier dysfunction may lead to abnormal urinary solutes migration, such as potassium, which depolarizes muscle and nerves, and then causes tissue injury and bladder pain $[9,10]$.

Histopathological studies of IC/BPS bladder have confirmed the involvement of macrophages, eosinophils, and mast cells in the urothelium. Involvement of eosinophils is also supported by increasing urinary eosinophil cationic protein in the urine cytology [22]. In the ESSIC type 3C IC/BPS patients, increased expression of $\mathrm{B}$ - and $\mathrm{T}$-cell markers, decreased expression of urothelial markers, focal lymphoid aggregates in the submucosa and higher urinary immunoglobulin concentration were found [16]. This evidence demonstrates that chronic inflammation could be a fundamental cause of the urothelial dysfunction in IC/BPS.

\section{CHRONIC INFLAMMATION AND APOPTOSIS}

Increased apoptosis cells and decreased proliferative cells were noted in IC bladder urothelium compared with the control group [5]. P38 mitogen-activated protein kinase might be an important mediator of the antiproliferative factor in the bladder urothelial cells [23]. Increased levels of the apoptotic signaling molecules, including Bax, cleaved caspase- 3 , and Bad were elevated in the IC/BPS bladder tissues [24]. The apoptosis in IC/ BPS bladders might be due to upregulation of inflammatory signals. Chronic inflammation in the suburothelium may inhibit normal urothelial basal cell proliferation and affect apical urothelium function. Acute bladder irritation such as bacterial infection increases afferent nerve activity and results in longterm plasticity, and then lowers the threshold for nociceptive and mechanoceptive sensation $[25,26]$. A rise in bladder nerve growth factor (NGF) after any insult to the bladder initiates signals that are transported to the dorsal root ganglion or spinal cord [27,28].

A previous study revealed abnormal differentiation in the urothelium of IC/BPS bladders with loss of E-cadherin and altered differentiation markers [8]. E-cadherin concentration in the IC/BPS bladder urothelium is significantly lower than that of healthy controls [29]. ZO-1 and E-cadherin expression was only decreased in IC/BPS, but not in patients with overactive bladder $(\mathrm{OAB})$, suggesting the barrier function of urothelium was prominent in IC/BPS but not altered in the OAB bladders [30]. However, decrease of E-cadherin expression is also associated with recurrent urinary tract infections in women [30-32]. These pathological differences between IC/BPS and other hypersensitive bladder diseases might result in distinct urothelial protein production. The pathophysiology of IC/BPS urothelium involves an aberrant differentiation program that results in altered synthesis of proteoglycans, tight junction proteins, cell adhesive proteins, and bacterial defense molecules such as GP51 [33-35]. Therefore, replacement therapy such as intravesical glycosaminoglycan instillation has been effectively used for treatment of IC/BPS [36].

\section{GLOMERULATIONS OF BLADDER UROTHELIUM IN IC/BPS}

IC bladders showed characteristic changes after hydrodistention under a certain pressure. Aberrant urothelial cells disrupt the permeability barrier, endothelial cell injury results in glomerulation bleeding, and degenerative change of nerves and regenerative features are frequently encountered in IC/BPS bladders $[37,38]$. Several inflammatory proteins were associated with increased angiogenesis and glomerulations in IC/BPS, including vascular endothelial growth factor (VEGF) [39], hypoxia-inducible factor-1-alpha [40], and platelet derived endothelial cell growth factor/thymidine phosphorylase [37]. Increased VEGF expression in the urothelium was associated with bladder inflammation and smaller bladder capacity in IC/ BPS patients. Down-regulation of VEGF in IC.BPS bladder tissue has been noted after repeated botulinum toxin A (BoNT-A) injections [41]. Measuring these angiogenic growth factors concentrations in the urine may be a new and useful method for the diagnosis of IC/BPS.

Previous studies have revealed that repeated intravesical BoNT-A injections could reduce bladder pain in patients with IC/BPS $[42,43]$. The elevated NGF levels in the IC/BPS bladder tissue significantly decreased to the normal range after BoNT- 
A treatment [44]. Repeated BoNT-A injections plus cystoscopic hydrodistention provide better therapeutic outcomes in treating IC/BPS than a single treatment [45]. Since repeated BoNTA injections could increase bladder capacity and relieve bladder pain in responders, the results provide evidence of urothelial repair and suburothelial inflammation reduction in the IC/BPS responders to BoNT-A. Chronic suburothelial inflammation might damage the urothelial function and cell differentiation, and repeated BoNT-A injections may gradually reduce the urothelium inflammation, thereby improving the symptoms in patients with IC/BPS [46].

\section{CENTRAL NERVOUS SENSITIZATION IN IC/BPS}

Recent evidence revealed neurogenic inflammation may play an essential role in the pathogenesis of several diseases, including arthritis, migraines, asthma, and possibly, IC/BPS [47]. Preliminary studies have shown the levels of immunoreactive substance $\mathrm{P}$ and NGF increase in the bladder tissue and urine of IC/BPS patients $[48,49]$. Increase of urinary adenosine triphosphate (ATP) and increased stretch-activated ATP release by bladder urothelial cells have been found in IC/BPS patients, suggesting augmented purinergic signaling in IC/BPS bladders [50]. Insult to the bladder wall or urothelium might induce inflammatory reactions and subsequently produce painful inflammation, such as in IC/BPS [51].

The chronic pain in IC/PBS may also result from CNS sensitization and persisting activation of the afferent sensory system in the urinary bladder [52]. Central c-fos expression was increased in the rat models of neurogenic detrusor overactivity and chronic inflammation [53]. Suppressing the NGF levels in spinal cord dorsal root ganglia could suppress bladder hyperreflexia [54]. Mechanical stimulus in IC/BPS patients induced a significantly higher stimulus-response curve, and a segmental hyperalgesia was noted, suggesting spinal central sensitization was involved in the IC/BPS pathophysiology [55]. If the neurogenic inflammation in the DGR or CNS can be reduced gradually after treatment, the visceral pain in IC/PBS may be relieved.

\section{PROTEOMICS INVESTIGATION OF THE POTENTIAL SERUM AND URINARY BIOMARKERS}

In recent years, the lamina propria of the bladder has been found to play a crucial role in transmitting the bladder sensa- tion in response to chemical stimuli and inflammation of the bladder [56,57]. Increased NGF levels in the urothelium and urine have been reported in patients with IC/BPS [58,59]. However, urinary NGF levels are also elevated in several lower urinary tract diseases such as bladder outlet obstruction, urinary tract stones, $\mathrm{OAB}$, and urinary tract infection [59]. Nevertheless, the level of urinary NGF is closely related to the visual analog pain scale and decreases in responders to conventional treatment for IC/BPS [60]. Urinary NGF levels were significantly decreased in and associated with greater pain improvement and a successful response to intravesical BoNT-A injections, suggesting urinary NGF, and although not specific to IC/ BPS, might be a useful biomarker for measuring the bladder condition severity in IC/BPS patients.

In addition, abnormal cell differentiation in the IC/BPS bladder urothelium was remarkable, which causes alteration of several barrier proteins and junction protein production. It is possible to search for possible urinary biomarkers for early detection of IC/BPS in patients with frequency urgency syndrome [61]. A previous study showed the urinary levels of inflammatory cytokines were elevated in patients with IC/BPS, including IL-2, IL-6, and IL-8 [62], but not specific to National Institute of Diabetes, Digestive and Kidney Diseases (NIDDK) defined IC/ BPS patients [63]. Although cytokine urinary level alone might not be useful to make a diagnosis of IC/BPS, a combination of several different urinary cytokines might raise the diagnostic rate of IC/BPS (Table 2).

Bladder biopsies from IC/BPS patients have confirmed the presence of macrophages and eosinophils in the urothelium and increased mast cell count in the detrusor [1,37]. In cyclophosphamide cystitis rats, elevated levels of chemokines and activation of mast cells were noted [64]. The most important inflammatory mediators causing chemotactic migration of mast cells, eosinophils and macrophages include MCP-1 (CCL2), macrophage inflammatory protein (MIP-1 $\beta$ ) (CCL4), and eotaxin (CCL11) [65-67]. Chemokines may also directly trigger these target cells in the bladder and thereby induce the inflammatory changes in IC/BPS. Analysis of multiple proteins from a urine sample is a convenient approach to measuring the activation of inflammatory cells in the bladder tissue [68].

Elevated serum C-reactive protein (CRP) has been noted in IC/BPS patients [69]. Serum CRP levels might be used to distinguish patients with IC/BPS from bladder hypersensitivity disorders. A previous study also revealed that the serum NGF level was elevated in IC/BPS patients [70]. However, there was 
Table 2. Potential urine and serum biomarkers for the diagnosis of IC/BPS

\begin{tabular}{|c|c|c|c|}
\hline Protein & Source & Evidence of activity & Reference \\
\hline \multirow[t]{2}{*}{ APF } & Urine & APF altered the pattern of cellular gene expression toward a phenotype like IC/BPS. & 9 \\
\hline & & APF treatment decreased expression of tight junction proteins $\mathrm{ZO}-1$ and occluding. & 10,11 \\
\hline HB-EGF & Urine & $\begin{array}{l}\text { Urine HB-EGF levels were significantly lower in IC/BPS patients. APF and HB-EGF levels were similar } \\
\text { in ulcerative and nonulcerative IC/BPS }\end{array}$ & 10 \\
\hline EGF & Urine & EGF levels were significantly higher in IC/BPS & 10 \\
\hline NGF & Urine & Increased NGF levels in IC/BPS and sensory urgency & 59 \\
\hline NGF & Urine & $\begin{array}{l}\text { A decrease of urinary NGF level was associated with greater pain reduction and a successful response to } \\
\text { treatment }\end{array}$ & 60 \\
\hline IL-2, IL-6, IL-8 & Urine & Significant elevations in symptom scores and IL-2, IL-6, and IL-8 were found in active IC/BPS & 62 \\
\hline ATP & Urine & Increased urinary ATP and increased stretch-activated ATP release by bladder urothelial cells & 50 \\
\hline Chemokines & Urine & A significant fivefold to twentyfold increase in CXCL-10 and 1, IL-6 and NGF in ulcer IC & 68 \\
\hline GP51 & Urine & Low urine GP51 levels in IC/BPS patients compared to normal controls & 35 \\
\hline CRP & Serum & Serum CRP levels were significantly higher in IC/BPS & 69 \\
\hline $\operatorname{IgE}$ & Serum & Elevated serum IgE level in IC/BPS & 20 \\
\hline NGF & Serum & Serum NGF was elevated in IC/BPS patients & 70 \\
\hline $\begin{array}{l}\text { IL- } 1 \beta, \text { IL- } 6 \text {, TNF- } \alpha \text {, } \\
\text { and IL- } 8\end{array}$ & Serum & $\begin{array}{l}\text { Serum proinflammatory cytokine and chemokine significantly higher in patients with IC/BPS than } \\
\text { controls }\end{array}$ & 71 \\
\hline
\end{tabular}

IC/BPS, interstitial cystitis/bladder pain syndrome; APF, antiproliferative factor; ZO-1, zonula occludens-1; HB-EGF, heparin-binding epidermal growth factor; EGF, epidermal growth factor; NGF, nerve growth factor; IL, interleukin; ATP, adenosine triphosphate; CXCL, CXC chemokine ligand; GP51, glycoprotein 51; CRP, C-reactive protein; TNF- $\alpha$, tumor necrosis factor- $\alpha$.

no significant correlation between the urinary and serum NGF levels in patients with IC/BPS. The serum NGF levels are not significantly different between IC/BPS patients with different clinical characteristics and comorbidities [70]. Cytokines and chemokines have been demonstrated to play critical roles in the pathogenesis of many chronic inflammatory diseases. The upregulated serum tumor necrosis factor- $\alpha$, IL-1 $\beta$, IL- 6 , and IL-8 levels in IC/BPS patients may potentially have a prognostic role and might help physicians choose an appropriate therapeutic agent. One recent study revealed these serum proinflammatory cytokines and chemokine levels were significantly higher in IC/ BPS patients than in the controls [71]. Increased expression of these proinflammatory cytokines and chemokine levels in the serum of IC/BPS patients suggests that not only mast cell activation, but also some other inflammatory mediators might play crucial roles in the pathogenesis of IC/BPS.

\section{ULTRASTRUCTURE INVESTIGATION OF THE UROTHELIAL CELLS IN IC/BPS BLADDER}

IC/BPS has been considered a disease of the bladder urotheli- um [72]. Urothelium leakage has been proposed as the cause which generates bladder symptoms of IC/BPS [14]. Bladder urothelium denudation and thinning, aberrant synthesis of proteoglycans, cell adhesive proteins and tight junction proteins, and bacterial defence molecules were found in the IC/ BPS bladders [33-35].

Three important components involved in bladder permeability barrier function are: apical membrane, tight junctions, and an active trafficking mechanism [73,74]. Umbrella cells located at the apical surface of bladder mucosa are the main cell layer that maintains the barrier function. Mature umbrella cells form uroplakins and well-defined tight junctions [75]. Loss of umbrella cells cause exposure of intermediate cells and loss of barrier function and active trafficking mechanism of the bladder epithelium $[74,76]$. Therefore, leakage of urine solutes into the underlying suburothelial layer results in sensory receptor exposure, so patients might develop irritative bladder symptoms and small functional bladder capacity [77]. Bacterial cystitis, mechanical trauma, and exposure to noxious chemicals lead to disruption of the bladder permeability barrier in IC/BPS [76,77]. Although a rapid recovery process will take place after urotheli- 
al damage, the maturation of the intermediate cells needs time and subsequent neurogenic inflammation might result in further damage to the bladder wall [78].

IC/BPS is a heterogeneous and progressive syndrome. $\mathrm{Pa}-$ tients with IC/BPS have bladder pain, urinary frequency, and urgency to varied degrees. After cystoscopic hydrodistention, the glomerulations developed vary from a mild to severe haemorrhage in appearance. The maximal bladder capacity also varies greatly. However, diffused bladder wall thickening and hydronephrosis which are frequently seen in patients with severe ketamine cystitis are not observed in IC/BPS bladders [79]. In microscopic histopathological examination of IC/BPS bladders, the epithelium denudation, inflammation with or without granulation tissue formation may present in the bladders in different degrees of severity. The IC bladders might have very mild to severely inflamed urothelium which causes symptoms from mild urinary frequency-urgency to severe bladder pain [15]. Therefore, understanding the urothelial ultrastructure changes in different stages of IC/BPS is very important for urologists for screening IC/BPS bladder of those patients with bladder hypersensitivity.

\section{MAPPING THE HETEROGENEITY OF IC/BPS}

IC/BPS is a clinical syndrome characterized by bladder pain, usually with urinary frequency, urgency, and nocturia. Different subtypes of IC/BPS have been identified, such as ulcer type and nonulcer type, which might have distinct clinical characteristics and underlying pathophysiology $[80,81]$. Pelvic pain is considered the hallmark symptom of IC/BPS patients, but patients with mild to moderate IC/BPS might only present with urinary frequency-urgency [82].

Classification of IC/BPS subtypes could be made with the findings of cystoscopy after hydrodistention and morphological findings in bladder biopsy [83]. This definition of IC/BPS by symptoms alone has been accepted by the American Urological Association [82], the European Association of Urology [83], as well as the International Consultation on Incontinence [84]. However, Asian urologists have proposed different diagnostic criteria of IC/BPS. For the definite diagnosis of IC/BPS, cystoscopy or hydrodistention is considered to be an essential diagnostic test by Eastern urologists, while hypersensitive bladder syndrome was used as the diagnosis if IC/BPS has not been confirmed by the required criteria $[3,4]$.

The NIDDK developed the research criteria for IC/BPS since
1987 [85]. However, the criteria are too stringent for clinical use [86]. The severity of cystoscopic findings after hydrodistention was not found to be significantly correlated with the degree of bladder inflammation [87]. Erickson et al. [63] found similar urine markers and bladder biopsy findings between 2 groups. Recently, we have revisited the diagnostic roles of the potassium sensitivity test (PST) and cystoscopic hydrodistention [88]. Both PST and glomerulations after hydrodistention are found to be sensitive indicators of IC/BPS, but the specificity of glomerulations is lower than that of PST in the diagnosis of IC/ BPS [88]. Therefore, construction of a diagnostic framework is needed for distinguishing IC/BPS from bladder disorders with similar symptoms. This diagnostic framework may facilitate early diagnosis and appropriate treatment of IC/BPS [89].

IC/BPS patients are also found to have higher odds of comorbid autoimmune or neurological diseases, such as rheumatological diseases and mental illnesses [90]. They also have higher risks of irritable bowel syndrome, fibromyalgia, general fatigue and functional somatic syndrome [91-94]. A distinct phenotype of IC/BPS patients with multiple sensitivities has been identified [95]. Pelvic morbidity is also related to symptom development. Women with IC/BPS often have a gynecologic disease history such as cesarean births, hysterectomy, miscarriage, stillbirth or abortion $[92,96]$. The symptoms in IC/ BPS women are also likely affected by the menstruation cycle [97]. Myofascial pain is often demonstrated in IC/BPS and suggested for therapeutic implication [98]. In patients with concurrent IC/BPS and irritable bowel syndrome, bladder symptoms will improve after treating small-intestinal bacterial overgrowth with antibiotics [99]. Cross-talk in pelvic organs has been suggested as a possible pathomechanism by which uterine or bowel pathology modulates the IC/BPS symptoms [100].

Investigation of the potential biomarkers of IC/BPS according to the possible pathophysiology has progressed greatly in recent decades. However, clinically applicable biomarkers in urinary or serum have not yet been well developed in the diagnosis of IC/BPS. Measuring urinary proteins or serum cytokines is a possible convenient approach to monitoring the inflammatory cell activity in the bladder tissue. Differences in urinary or serum biomarkers could provide a diagnostic basis for IC/BPS subtypes and might be a useful tool for the differential diagnosis among IC/BPS, hypersensitive bladder, and OAB. In addition, the involvement of IC/BPS in systemic functional somatic syndrome and other pelvic organ diseases might also subdivide another subtype of IC/BPS. Mapping the heterogene- 
ity of IC/BPS might also provide different treatment strategies and prognostic outcomes.

\section{CONCLUSION}

The pathophysiology of IC/BPS might involve chronic inflammation, increased apoptosis, urothelial dysfunction, and CNS sensitization. In addition, systemic involvement of IC/BPS with multiple comorbidities and functional somatic syndromes are also found together with increased sympathetic nervous system tonicity and increased serum proinflammatory proteins and cytokines. Through analyzing different presentations in bladder conditions, urothelial dysfunction, urinary biomarker production, and immunohistochemistry of the bladder wall, it is possible to map IC/BPS into different phenotypes and provide evidence for successful treatment.

\section{REFERENCES}

1. Sant GR, Kempuraj D, Marchand JE, Theoharides TC. The mast cell in interstitial cystitis: role in pathophysiology and pathogenesis. Urology 2007;69(4 Suppl):34-40.

2. Keay $S$. Cell signaling in interstitial cystitis/painful bladder syndrome. Cell Signal 2008;20:2174-9.

3. Homma Y, Ueda T, Tomoe H, Lin AT, Kuo HC, Lee MH, et al. Clinical guidelines for interstitial cystitis and hypersensitive bladder syndrome. Int J Urol 2009;16:597-615.

4. Homma Y, Ueda T, Tomoe H, Lin AT, Kuo HC, Lee MH, et al. Clinical guidelines for interstitial cystitis and hypersensitive bladder updated in 2015. Int J Urol 2016;23:542-9.

5. Shie JH, Kuo HC. Higher levels of cell apoptosis and abnormal Ecadherin expression in the urothelium are associated with inflammation in patients with interstitial cystitis/painful bladder syndrome. BJU Int 2011;108(2 Pt 2):E136-41.

6. Southgate J, Varley CL, Garthwaite MA, Hinley J, Marsh F, Stahlschmidt J, et al. Differentiation potential of urothelium from patients with benign bladder dysfunction. BJU Int 2007;99:150616.

7. Zeng Y, Wu XX, Homma Y, Yoshimura N, Iwaki H, Kageyama S, et al. Uroplakin III-delta4 messenger RNA as a promising marker to identify nonulcerative interstitial cystitis. J Urol 2007;178(4 Pt 1):1322-7.

8. Hauser PJ, Dozmorov MG, Bane BL, Slobodov G, Culkin DJ, Hurst RE. Abnormal expression of differentiation related proteins and proteoglycan core proteins in the urothelium of patients with interstitial cystitis. J Urol 2008;179:764-9.
9. Kim J, Keay SK, Dimitrakov JD, Freeman MR. p53 mediates interstitial cystitis antiproliferative factor (APF)-induced growth inhibition of human urothelial cells. FEBS Lett 2007;581:3795-9.

10. Zhang CO, Li ZL, Kong CZ. APF, HB-EGF, and EGF biomarkers in patients with ulcerative vs. non-ulcerative interstitial cystitis. BMC Urol 2005;5:7.

11. Keay S, Kaczmarek P, Zhang CO, Koch K, Szekely Z, Barchi JJ Jr, et al. Normalization of proliferation and tight junction formation in bladder epithelial cells from patients with interstitial cystitis/painful bladder syndrome by d-proline and d-pipecolic acid derivatives of antiproliferative factor. Chem Biol Drug Des 2011;77:421-30.

12. Tseng-Rogenski S, Liebert M. Interleukin-8 is essential for normal urothelial cell survival. Am J Physiol Renal Physiol 2009;297:F81621.

13. Graham E, Chai TC. Dysfunction of bladder urothelium and bladder urothelial cells in interstitial cystitis. Curr Urol Rep 2006;7:440-6.

14. Parsons CL. The role of a leaky epithelium and potassium in the generation of bladder symptoms in interstitial cystitis/overactive bladder, urethral syndrome, prostatitis and gynaecological chronic pelvic pain. BJU Int 2011;107:370-5.

15. Jhang JF, Hsu YH, Kuo HC. Characteristics and electrocauterization of Hunner's lesions associated with bladder pain syndrome. Urol Sci 2013; 24:51-5.

16. Gamper M, Viereck V, Eberhard J, Binder J, Moll C, Welter J, et al. Local immune response in bladder pain syndrome/interstitial cystitis ESSIC type 3C. Int Urogynecol J 2013;24:2049-57.

17. Homma Y, Nomiya A, Tagaya M, Oyama T, Takagaki K, Nishimat$\mathrm{su} \mathrm{H}$, et al. Increased mRNA expression of genes involved in pronociceptive inflammatory reactions in bladder tissue of interstitial cystitis. J Urol 2013;190:1925-31.

18. Colaco M, Koslov DS, Keys T, Evans RJ, Badlani GH, Andersson $\mathrm{KE}$, et al. Correlation of gene expression with bladder capacity in interstitial cystitis/bladder pain syndrome. J Urol 2014;192:1123-9.

19. Ogawa T, Homma T, Igawa Y, Seki S, Ishizuka O, Imamura T, et al. CXCR3 binding chemokine and TNFSF14 over expression in bladder urothelium of patients with ulcerative interstitial cystitis. J Urol 2010;183:1206-12.

20. Lee J, Doggweiler-Wiygul R, Kim S, Hill BD, Yoo TJ. Is interstitial cystitis an allergic disorder?: a case of interstitial cystitis treated successfully with anti-IgE. Int J Urol 2006;13:631-4.

21. Parsons CL. The role of the urinary epithelium in the pathogenesis of interstitial cystitis/prostatitis/urethritis. Urology 2007;69(4 Suppl):9-16.

22. Bouchelouche K, Kristensen B, Nordling J, Horn T, Bouchelouche P. Increased urinary leukotriene $\mathrm{E} 4$ and eosinophil protein $\mathrm{X}$ ex- 
cretion in patients with interstitial cystitis. J Urol 2001;166:2121-5.

23. Kim J, Keay SK, Freeman MR. Heparin-binding epidermal growth factor-like growth factor functionally antagonizes interstitial cystitis antiproliferative factor via mitogen-activated protein kinase pathway activation. BJU Int 2009;103:541-6.

24. Shie JH, Liu HT, Kuo HC. Increased cell apoptosis of urothelium mediated by inflammation in interstitial cystitis/painful bladder syndrome. Urology 2012;79:484.e7-13.

25. Murray E, Malley SE, Qiao LY, Hu VY, Vizzard MA. Cyclophosphamide induced cystitis alters neurotrophin and receptor tyrosine kinase expression in pelvic ganglia and bladder. J Urol 2004;172(6 Pt 1):2434-9.

26. Oddiah D, Anand P, McMahon SB, Rattray M. Rapid increase of NGF, BDNF and NT-3 mRNAs in inflamed bladder. Neuroreport 1998;9:1455-8.

27. Chuang YC, Fraser MO, Yu Y, Chancellor MB, de Groat WC, Yoshimura N. The role of bladder afferent pathways in bladder hyperactivity induced by the intravesical administration of nerve growth factor. J Urol 2001;165:975-9.

28. Lamb K, Gebhart GF, Bielefeldt K. Increased nerve growth factor expression triggers bladder overactivity. J Pain 2004;5:150-6.

29. Lee CL, Jiang YH, Kuo HC. Increased apoptosis and suburothelial inflammation in patients with ketamine-related cystitis: a comparison with non-ulcerative interstitial cystitis and controls. BJU Int 2013;112:1156-62.

30. Liu HT, Shie JH, Chen SH, Wang YS, Kuo HC. Differences in mast cell infiltration, E-cadherin, and zonula occludens-1 expression between patients with overactive bladder and interstitial cystitis/bladder pain syndrome. Urology 2012;80:225.e13-8.

31. van Roy F, Berx G. The cell-cell adhesion molecule E-cadherin. Cell Mol Life Sci 2008;65:3756-88.

32. Chuang FC, Kuo HC. Increased urothelial cell apoptosis and chronic inflammation are associated with recurrent urinary tract infection in women. PLoS One 2013;8:e63760.

33. Slobodov G, Feloney M, Gran C, Kyker KD, Hurst RE, Culkin DJ. Abnormal expression of molecular markers for bladder impermeability and differentiation in the urothelium of patients with interstitial cystitis. J Urol 2004;171:1554-8.

34. Min G, Zhou G, Schapira M, Sun TT, Kong XP. Structural basis of urothelial permeability barrier function as revealed by Cryo-EM studies of the $16 \mathrm{~nm}$ uroplakin particle. J Cell Sci 2003;116(Pt 20):4087-94.

35. Byrne DS, Sedor JF, Estojak J, Fitzpatrick KJ, Chiura AN, Mulholland SG. The urinary glycoprotein GP51 as a clinical marker for interstitial cystitis. J Urol 1999;161:1786-90.
36. Hurst RE, Moldwin RM, Mulholland SG. Bladder defense molecules, urothelial differentiation, urinary biomarkers, and interstitial cystitis. Urology 2007;69(4 Suppl):17-23.

37. Van De Merwe JP, Arendsen HJ. Interstitial cystitis: a review of immunological aspects of the aetiology and pathogenesis, with a hypothesis. BJU Int 2000;85:995-9.

38. Saban R, Saban MR, Maier J, Fowler B, Tengowski M, Davis CA, et al. Urothelial expression of neuropilins and VEGF receptors in control and interstitial cystitis patients. Am J Physiol Renal Physiol 2008;295:F1613-23.

39. Lee JD, Lee MH. Increased expression of hypoxia-inducible factor$1 \alpha$ and vascular endothelial growth factor associated with glomerulation formation in patients with interstitial cystitis. Urology 2011;78:971.e11-5.

40. Tamaki M, Saito R, Ogawa O, Yoshimura N, Ueda T. Possible mechanisms inducing glomerulations in interstitial cystitis: relationship between endoscopic findings and expression of angiogenic growth factors. J Urol 2004;172:945-8.

41. Peng $\mathrm{CH}$, Jhang JF, Shie JH, Kuo HC. Down regulation of vascular endothelial growth factor is associated with decreased inflammation after intravesical OnabotulinumtoxinA injections combined with hydrodistention for patients with interstitial cystitis--clinical results and immunohistochemistry analysis. Urology 2013;82:1452. e1-6.

42. Kuo HC. Preliminary results of suburothelial injection of botulinum a toxin in the treatment of chronic interstitial cystitis. Urol Int 2005;75:170-4.

43. Giannantoni A, Costantini E, Di Stasi SM, Tascini MC, Bini V, Porena M. Botulinum A toxin intravesical injections in the treatment of painful bladder syndrome: a pilot study. Eur Urol 2006;49:704-9.

44. Liu HT, Kuo HC. Intravesical botulinum toxin A injections plus hydrodistension can reduce nerve growth factor production and control bladder pain in interstitial cystitis. Urology 2007;70:463-8.

45. Lee CL, Kuo HC. Long-term efficacy and safety of repeated intravescial onabotulinumtoxinA injections plus hydrodistention in the treatment of interstitial cystitis/bladder pain syndrome. Toxins (Basel) 2015;7:4283-93.

46. Shie JH, Liu HT, Wang YS, Kuo HC. Immunohistochemical evidence suggests repeated intravesical application of botulinum toxin A injections may improve treatment efficacy of interstitial cystitis/ bladder pain syndrome. BJU Int 2013;111:638-46.

47. Elbadawi A. Interstitial cystitis: a critique of current concepts with a new proposal for pathologic diagnosis and pathogenesis. Urology 1997;49(5A Suppl):14-40. 
48. Steers WD, Tuttle JB. Neurogenic inflammation and nerve growth factor: possible roles in interstitial cystitis. In: Sant GR, editor. Interstitial cystitis. Philadelphia: Lippincott-Raven Publishers; 1997. p. 67-75.

49. Lowe EM, Anand P, Terenghi G, Williams-Chestnut RE, Sinicropi DV, Osborne JL. Increased nerve growth factor levels in the urinary bladder of women with idiopathic sensory urgency and interstitial cystitis. Br J Urol 1997;79:572-7.

50. Sun Y, Chai TC. Augmented extracellular ATP signaling in bladder urothelial cells from patients with interstitial cystitis. Am J Physiol Cell Physiol 2006;290:C27-34.

51. Wesselmann U. Interstitial cystitis: a chronic visceral pain syndrome. Urology 2001;57(6 Suppl 1):32-9.

52. Steers WD, Tuttle JB. Mechanisms of disease: the role of nerve growth factor in the pathophysiology of bladder disorders. Nat Clin Pract Urol 2006;3:101-10.

53. Cui M, Khanijou S, Rubino J, Aoki KR. Subcutaneous administration of botulinum toxin A reduces formalin-induced pain. Pain 2004;107:125-33.

54. Seki S, Sasaki K, Fraser MO, Igawa Y, Nishizawa O, Chancellor MB, et al. Immunoneutralization of nerve growth factor in lumbosacral spinal cord reduces bladder hyperreflexia in spinal cord injured rats. J Urol 2002;168:2269-74.

55. Lai HH, Gardner V, Ness TJ, Gereau RW 4th. Segmental hyperalgesia to mechanical stimulus in interstitial cystitis/bladder pain syndrome: evidence of central sensitization. J Urol 2014;191:1294-9.

56. Wiseman OJ, Fowler CJ, Landon DN. The role of the human bladder lamina propria myofibroblast. BJU Int 2003;91:89-93.

57. Avelino A, Cruz C, Nagy I, Cruz F. Vanilloid receptor 1 expression in the rat urinary tract. Neuroscience 2002;109:787-98.

58. Dupont MC, Spitsbergen JM, Kim KB, Tuttle JB, Steers WD. Histological and neurotrophic changes triggered by varying models of bladder inflammation. J Urol 2001;166:1111-8.

59. Kuo HC, Liu HT, Tyagi P, Chancellor MB. Urinary nerve growth factor levels in urinary tract diseases with or without frequency urgency symptoms. Low Urin Tract Symptoms 2010;2:88-94.

60. Liu HT, Tyagi P, Chancellor MB, Kuo HC. Urinary nerve growth factor level is increased in patients with interstitial cystitis/bladder pain syndrome and decreased in responders to treatment. BJU Int 2009;104:1476-81.

61. Wilkinson DR, Erickson AD. Urinary and serologic markers forinterstitial cystitis: an update. Curr Urol Rep 2006;7:414-22.

62. Peters KM, Diokno AC, Steinert BW. Preliminary study on urinary cytokine levels in interstitial cystitis: does intravesical bacille Calmette-Guérin treat interstitial cystitis by altering the immune profile in the bladder? Urology 1999;54:450-3.

63. Erickson DR, Tomaszewski JE, Kunselman AR, Bentley CM, Peters KM, Rovner ES, et al. Do the National Institute of Diabetes and Digestive and Kidney Diseases cystoscopic criteria associate with other clinical and objective features of interstitial cystitis? J Urol 2005;173:93-7.

64. Smaldone MC, Vodovotz Y, Tyagi V, Barclay D, Philips BJ, Yoshimura $\mathrm{N}$, et al. Multiplex analysis of urinary cytokine levels in rat model of cyclophosphamide-induced cystitis. Urology 2009;73:4216.

65. Bouchelouche K, Alvarez S, Andersen L, Nordling J, Horn T, Bouchelouche P. Monocyte chemoattractant protein-1 production by human detrusor smooth muscle cells. J Urol 2004;171:462-6.

66. Lintomen L, Franchi G, Nowill A, Condino-Neto A, de Nucci G, Zanesco A, et al. Human eosinophil adhesion and degranulation stimulated with eotaxin and RANTES in vitro: lack of interaction with nitric oxide. BMC Pulm Med 2008;8:13.

67. Muessel MJ, Scott KS, Friedl P, Bradding P, Wardlaw AJ. CCL11 and GM-CSF differentially use the Rho GTPase pathway to regulate motility of human eosinophils in a three-dimensional microenvironment. J Immunol 2008;180:8354-60.

68. Tyagi P, Killinger K, Tyagi V, Nirmal J, Chancellor M, Peters KM. Urinary chemokines as noninvasive predictors of ulcerative interstitial cystitis. J Urol 2012;187:2243-8.

69. Chung SD, Liu HT, Lin H, Kuo HC. Elevation of serum c-reactive protein in patients with $\mathrm{OAB}$ and IC/BPS implies chronic inflammation in the urinary bladder. Neurourol Urodyn 2011;30:417-20.

70. Liu HT, Kuo HC. Increased urine and serum nerve growth factor levels in interstitial cystitis suggest chronic inflammation is involved in the pathogenesis of disease. PLoS One 2012;7:e44687.

71. Jiang YH, Peng CH, Liu HT, Kuo HC. Increased pro-inflammatory cytokines, C-reactive protein and nerve growth factor expressions in serum of patients with interstitial cystitis/bladder pain syndrome. PLoS One 2013;8:e76779.

72. Messing EM, Stamey TA. Interstitial cystitis: early diagnosis, pathology, and treatment. Urology 1978;12:381-92.

73. Negrete HO, Lavelle JP, Berg J, Lewis SA, Zeidel ML. Permeability properties of the intact mammalian bladder epithelium. Am J Physiol 1996;271(4 Pt 2):F886-94.

74. Zeidel ML. Low permeabilities of apical membranes of barrier epithelia: what makes watertight membranes watertight? Am J Physiol 1996;271(2 Pt 2):F243-5.

75. Tzan CJ, Berg J, Lewis SA. Effect of protamine sulfate on the permeability properties of the mammalian urinary bladder. J Membr Biol 1993;133:227-42. 
76. Lewis SA, Berg JR, Kleine TJ. Modulation of epithelial permeability by extracellular macromolecules. Physiol Rev 1995;75:561-89.

77. Lavelle JP, Meyers SA, Ruiz WG, Buffington CA, Zeidel ML, Apodaca G. Urothelial pathophysiological changes in feline interstitial cystitis: a human model. Am J Physiol Renal Physiol 2000;278:F540-53.

78. Lavelle J, Meyers S, Ramage R, Bastacky S, Doty D, Apodaca G, et al. Bladder permeability barrier: recovery from selective injury of surface epithelial cells. Am J Physiol Renal Physiol 2002;283:F24253.

79. Huang LK, Wang JH, Shen SH, Lin AT, Chang CY. Evaluation of the extent of ketamine-induced uropathy: the role of CT urography. Postgrad Med J 2014;90:185-90.

80. Bouchelouche K, Nordling J. Recent developments in the management of interstitial cystitis. Curr Opin Urol 2003;13:309-13.

81. Hanno PM, Sant GR. Clinical highlights of the National Institute of Diabetes and Digestive and Kidney Diseases/Interstitial Cystitis Association scientific conference on interstitial cystitis. Urology 2001;57(6 Suppl 1):2-6.

82. Hanno PM, Burks DA, Clemens JQ, Dmochowski RR, Erickson D, Fitzgerald MP, et al. AUA guideline for the diagnosis and treatment of interstitial cystitis/bladder pain syndrome. J Urol 2011;185:216270 .

83. van de Merwe JP, Nordling J, Bouchelouche P, Bouchelouche K, Cervigni M, Daha LK, et al. Diagnostic criteria, classification, and nomenclature for painful bladder syndrome/interstitial cystitis: an ESSIC proposal. Eur Urol 2008;53:60-7.

84. Hanno P, Lin A, Nordling J, Nyberg L, van Ophoven A, Ueda T, et al. Bladder Pain Syndrome Committee of the International Consultation on Incontinence. Neurourol Urodyn 2010;29:191-8.

85. Gillenwater JY, Wein AJ. Summary of the National Institute of Arthritis, Diabetes, Digestive and Kidney Diseases Workshop on Interstitial Cystitis, National Institutes of Health, Bethesda, Maryland, August 28-29, 1987. J Urol 1988;140:203-6.

86. Hanno PM, Landis JR, Matthews-Cook Y, Kusek J, Nyberg L Jr. The diagnosis of interstitial cystitis revisited: lessons learned from the National Institutes of Health Interstitial Cystitis Database study. J Urol 1999;161:553-7.

87. Denson MA, Griebling TL, Cohen MB, Kreder KJ. Comparison of cystoscopic and histological findings in patients with suspected interstitial cystitis. J Urol 2000;164:1908-11.

88. Jiang YH, Jhang JF, Kuo HC. Revisiting the role of potassium sensitivity testing and cystoscopic hydrodistention for the diagnosis of interstitial cystitis. PLoS One 2016;11:e0151692.
89. Macdiarmid SA, Sand PK. Diagnosis of interstitial cystitis/painful bladder syndrome in patients with overactive bladder symptoms. Rev Urol 2007;9:9-16.

90. Keller JJ, Chen YK, Lin HC. Comorbidities of bladder pain syndrome/interstitial cystitis: a population-based study. BJU Int 2012; 110(11 Pt C):E903-9.

91. Martínez-Martínez LA, Mora T, Vargas A, Fuentes-Iniestra M, Martínez-Lavín M. Sympathetic nervous system dysfunction in fibromyalgia, chronic fatigue syndrome, irritable bowel syndrome, and interstitial cystitis: a review of case-control studies. J Clin Rheumatol 2014;20:146-50.

92. Warren JW, Morozov V, Howard FM, Wesselmann U, Gallicchio L, Langenberg $\mathrm{P}$, et al. Before the onset of interstitial cystitis/bladder pain syndrome, the presence of multiple non-bladder syndromes is strongly associated with a history of multiple surgeries. J Psychosom Res 2014;76:75-9.

93. Nickel JC, Tripp DA; International Interstitial Cystitis Study Group. Clinical and psychological parameters associated with pain pattern phenotypes in women with interstitial cystitis/bladder pain syndrome. J Urol 2015;193:138-44.

94. Clemens JQ, Elliott MN, Suttorp M, Berry SH. Temporal ordering of interstitial cystitis/bladder pain syndrome and non-bladder conditions. Urology 2012;80:1227-31.

95. Fuoco MB, Irvine-Bird K, Curtis Nickel J. Multiple sensitivity phenotype in interstitial cystitis/bladder pain syndrome. Can Urol Assoc J 2014;8:E758-61.

96. Hall SA, Link CL, Pulliam SJ, Hanno PM, Eggers PW, Kusek JW, et al. The relationship of common medical conditions and medication use with symptoms of painful bladder syndrome: results from the Boston area community health survey. J Urol 2008;180:593-8.

97. Peters KM, Carrico DJ, Diokno AC. Characterization of a clinical cohort of 87 women with interstitial cystitis/painful bladder syndrome. Urology 2008;71:634-40.

98. Bassaly R, Tidwell N, Bertolino S, Hoyte L, Downes K, Hart S. Myofascial pain and pelvic floor dysfunction in patients with interstitial cystitis. Int Urogynecol J 2011;22:413-8.

99. Weinstock LB, Klutke CG, Lin HC. Small intestinal bacterial overgrowth in patients with interstitial cystitis and gastrointestinal symptoms. Dig Dis Sci 2008;53:1246-51.

100. Rudick CN, Chen MC, Mongiu AK, Klumpp DJ. Organ cross talk modulates pelvic pain. Am J Physiol Regul Integr Comp Physiol 2007;293:R1191-8. 